\title{
REPRESENTACIONES DEL ESTATUS SOCIAL EN ESTUDIANTES DEL ÚLTIMO AÑO DE EDUCACIÓN SECUNDARIA EN UN COLEGIO PRIVADO DE LIMA METROPOLITANA
}

\author{
FERNANDO GARCÍA \\ https://orcid.org/0000-0002-2120-514X \\ Universidad Autónoma de Barcelona / Barcelona, España \\ Recibido: 3 de febrero del 2020 / Aceptado: 23 de marzo del 2020 \\ doi: 10.26439/persona2020.n023(1).4835
}

\begin{abstract}
Resumen. El estatus social tiene un rol central en la vida del adolescente en la escuela. El presente estudio cualitativo busca analizar las representaciones del estatus social escolar en 10 estudiantes $(M=15,89, D E=0,33)$ del último año de educación secundaria de un colegio privado de Lima Metropolitana. Se aplicaron grupos focales y entrevistas a profundidad. Los resultados evidencian que la dominancia y el prestigio son estrategias efectivas para ganar estatus en la escuela. Los estudiantes dominantes muestran conductas agresivas, fortaleza física y están asociados con el consumo de drogas entre sus pares; los estudiantes prestigiosos se caracterizan por el éxito académico, la conducta prosocial y logros deportivos. Se halló también que la identidad no heterosexual y ser diagnosticado con un trastorno mental afectan negativamente el estatus social en la escuela. Se discuten las relaciones entre estos factores y sus posibles implicancias.
\end{abstract}

Palabras clave: estatus social / dominancia / prestigio / educación secundaria

\section{SOCIAL STATUS REPRESENTATIONS AMONG SENIOR HIGH SCHOOL STUDENTS FROM A PRIVATE SCHOOL IN LIMA METROPOLITAN AREA}

\footnotetext{
Abstract. Social status plays a central role in teenage school life. This qualitative study aims to examine the representations of school social status among 10 senior high school students $(M=15.89, S D=0.33)$ from a private school in Lima Metropolitan Area. Focus groups and in-depth interviews were conducted. The results evidence both dominance
}

Correo electrónico: Fernando.GarciaBl@e-campus.uab.cat 
and prestige are effective strategies to gain status at school. Dominant students show aggressive behaviors, physical strength, and are associated with drug use among their peers. Prestigious students' traits are academic achievement, prosocial behavior and sport success. Non-heterosexual identity and mental illness negatively affect social status in high school. The relationship between these factors and their possible implications is discussed.

Keywords: social status / dominance / prestige / secondary education 


\section{INTRODUCCIÓN}

La vida social involucra jerarquías que diferencian a los individuos según diversos criterios, como el acceso a recursos, el grado de influencia y el reconocimiento que unos sujetos tienen sobre otros (Fiske, Dupree, Nicolas y Swencionis, 2016). El estatus social influye en la manera como interactuamos, recordamos y evaluamos a los demás (Mattan, Kubota y Cloutier, 2017). Además, puede ser un eje de conflicto entre diferentes grupos sociales (Fiske et al., 2016).

La escuela, por su parte, es un espacio de socialización cuyo rol es protagónico en la vida de los alumnos. Diversos estudios han hallado relaciones positivas entre el estatus social alto y la salud (Mattan et al., 2017; Sweeting y Hunt, 2014), y el estatus familiar bajo con estrés y síntomas asociados a problemas de salud física (Rahal et al., 2019; Sweeting y Hunt, 2014) en adolescentes. En este sentido, el estatus tiene un impacto social, psicológico y fisiológico en este grupo. El presente estudio busca analizar las representaciones del estatus en adolescentes de último año de educación secundaria en un colegio privado de Lima Metropolitana.

\section{Estatus}

Las aproximaciones clásicas al estatus social son la teoría basada en la competencia y la teoría basada en el conflicto (Cheng, Tracy, Foulsham, Kingstone y Henrich, 2013). El estatus se define como una jerarquía de recompensas o de exhibiciones, o ambas al mismo tiempo. La primera sostiene que el rango social de un individuo es producto del consenso grupal sobre el valor que tiene para el colectivo, utilizando como criterios la experticia y la competencia superior en los dominios valorados socialmente. Por el contrario, la teoría basada en el conflicto afirma que las pruebas de dominancia y la función coercitiva son los sistemas fundamentales para jerarquizar a los individuos en un grupo: amenazas, miedos y diversas formas de agresión (Cheng et al., 2013).

Una aproximación más contemporánea es la teoría de dominancia-prestigio, desarrollada desde la psicología evolucionista por Henrich y Gil-White (2001), y aplicada en investigaciones recientes (Cheng et al., 2013; Kakkar, Sivanathan y Gobel, 2019; Kakkar y Sivanathan, 2017; Redhead, Cheng, Driver, Foulsham y 0'Gorman, 2018; Roberts, Palermo y Visser, 2019; Panchal y Gill, 2019; Witkower, Tracy, Cheng y Henrich, 2020). Esta perspectiva, que será utilizada en la presente investigación, define el estatus como el grado de deferencia, influencia, atención social y acceso a recursos valorados que recibe uno o más individuos de manera relativamente incuestionada (Cheng et al., 2013). Considera que hay dos caminos que implican procesos psicológicos distintos para alcanzar un mayor rango social en las sociedades humanas: la dominancia y el prestigio. 
La dominancia alude a la inducción del miedo a través de la intimidación y la coerción, similar a la teoría basada en el conflicto. Este criterio está presente también en especies no humanas, pues tiene su origen en la competencia agónica por recursos materiales imprescindibles para la supervivencia, que probablemente emergió para mantener los patrones de sumisión de los subordinados hacia los dominantes (Cheng et al., 2013). El prestigio, por otro lado, se refiere al rango social adquirido por individuos reconocidos y valorados por sus habilidades, éxitos o conocimientos (Henrich y Gil-White, 2001). Es la segunda vía para competir y alcanzar estatus, exclusiva de la especie humana, que resulta de la vida grupal y de las capacidades de aprendizaje social. Esto se debe a la evolución de la capacidad cultural, que creó un nuevo ambiente de selección que favorece las mutaciones que mejoran la transmisión de la información social de calidad, estable y confiable. Los individuos que tienen estas habilidades son modelos muy valorados y reciben deferencias de parte del resto de integrantes, que los copian (Cheng et al., 2013).

Ambas rutas son estrategias psicológicas desplegadas en ciertas situaciones, que involucran patrones emocionales, cognitivos, conductuales y motivacionales, orientados a resultados específicos. En esta línea, tales estrategias pueden coexistir simultáneamente en el mismo sujeto, dependiendo de quién juzgue (Cheng et al., 2013). Por ejemplo, un jefe puede ser querido por su experticia profesional y, al mismo tiempo, temido por su capacidad coercitiva. Como parte de la competencia por el estatus, los miembros del grupo que practican actos típicos de personas con un estatus superior podrían inducir a otros miembros a actuar con patrones típicos de personas con bajo estatus (Bianchi, Lancianese y Hunter, 2006).

En el campo escolar, se sugiere que conductas como el bullying son instrumentales, pues son medios que aumentan el estatus (Faris y Felmlee, 2009) a través de la ruta de la dominancia, para la teoría. Según estos autores, la agresión es un mecanismo de ascenso en la mayoría de los adolescentes, con excepción de los extremos de la jerarquía social, pues los que están en la cima no necesitan usarla y los que están en los niveles más bajos no tienen los recursos para hacerlo (Faris y Felmlee, 2011), por la ruta de la dominancia.

\section{Representaciones}

Las representaciones son, considerando su definición amplia, objetos mentales con propiedades semánticas que la mente almacena, construye y transforma mediante diversos procesos (Pitt, 2012). En este sentido, el concepto representación es central para entender cómo funciona la mente y ha sido abordado desde diferentes teorías.

Para Searle (1995), hay elementos constitutivos de la sociedad, como el estatus, que se codifican de manera automática, lo que implica que los seres humanos contamos con 
una estructura mental que nos hace posible entenderlas, interpretarlas y practicarlas. Searle (1995) afirma que esta estructura está compuesta por un conjunto de habilidades (background abilities) no intencionales o preintencionales que permiten las funcionesestatus. Las capacidades son el conjunto de tendencias, habilidades, disposiciones y estructuras causales en general, en el nivel neurofisiológico, que nos vemos forzados a explicar en niveles superiores por el poco conocimiento que tenemos sobre ellas actualmente. Se utiliza el verbo permitir porque las capacidades funcionan de manera causal en la producción de ciertos fenómenos intencionales. Estas habilidades actúan de manera generalizada, pues nos permiten superar el nivel de interpretación literal y pasar a un nivel más abstracto, socialmente construido, que implica un conocimiento del funcionamiento del mundo. Estos mecanismos han sido abordados desde la sociología con el concepto de habitus en Bourdieu y de realismo corporal en Shilling (Henry, 2013).

Las background abilities en Searle (1995) son todos los mecanismos automáticos que caracterizan al sistema 1 (S1) propuesto por Daniel Kahneman (2013), la teoría que utiliza el presente estudio. En su propuesta bimodal de los procesos cognitivos plantea, con fines descriptivos, que la mente funciona con dos sistemas: S1 y S2. El primero, como ya se ha mencionado, abarca todos los mecanismos encargados de los procesos y actividades automáticas. Por otro lado, S2 comprende el procesamiento y ejecución bajo el control de la conciencia; por lo tanto, se encarga de las acciones complejas que requieren esfuerzo cognitivo. Ambos funcionan para economizar el gasto de recursos cognitivos, pues el esfuerzo mental es aversivo para el cerebro porque consume glucosa (Kahneman, 2013).

S1 agrupa todos los mecanismos rápidos que demandan poco esfuerzo mental, habilidades innatas, conductas automatizadas, patrones automáticos de acción, recuperación de recuerdos e ideas, una parte de la memoria asociativa, las experiencias de cotidianidad, las impresiones de causalidad, sesgos e intuiciones. Sobre las últimas, Kahneman (2013) afirma que la intuición no es sino la recuperación automática de una idea que fue almacenada de la misma manera, por lo que la mente tiene mucha más información de lo que S2 cree que tiene. S1 es heurística, aplica rutas cognitivas que implican poco esfuerzo mental para resolver un problema.

Para S1, verdad es familiaridad, es decir, las experiencias e ideas que se repiten con alta frecuencia son consideradas verdaderas; la familiaridad de una frase en un enunciado puede hacer que todo su contenido parezca familiar. Kahneman (2013) propone el principio WYSIATI: "What you see is all there is", en español: "Lo que ves es todo lo que hay", que explica por qué la mente esboza conclusiones de manera incuestionada solo con la información disponible. 


\section{MÉTODO}

\section{Diseño de estudio}

La investigación es de tipo cualitativo, de nivel exploratorio-descriptivo y de método fenomenológico (Hernández, Fernández y Baptista, 2014). Este diseño nos permitirá entender los factores implicados en la construcción de significados que emergen de los fenómenos intersubjetivos a través del registro de las representaciones y experiencias (Berg, 2008). Asimismo, utiliza el método fenomenológico, debido a que el concepto de representación alude no al fenómeno per se, sino a cómo este es vivido por el sujeto (Vieytes, 2009).

\section{Técnicas e instrumentos de recolección de datos}

En el presente trabajo, se utilizó la entrevista a profundidad y la entrevista de grupos focales según las características de cada participante. Esta última nos permite explorar un fenómeno desde la construcción de conocimiento intersubjetivo de los participantes como conjunto (Dilshad y Latif, 2013), que empieza con el intercambio de ideas y termina en la conceptualización de significados compartidos (Morgan, 2012; Kornblit, Verardi y Beltramino, 2009). Se aplicó también la entrevista a profundidad con los dos participantes restantes, pues esta técnica se adaptó mejor a sus características personales y, de esta manera, obtuvimos información de mayor calidad. La entrevista a profundidad es una entrevista personal no directiva que sitúa al entrevistador en una posición de neutralité bienveillante, que consiste en la capacidad de validar las respuestas del participante de manera amable y sin juzgarla (Berthier, 2006), lo que promueve la expresión autorreflexiva del sujeto en un espacio emocionalmente seguro (Kornblit et al., 2009); por eso es ideal para abordar temas personales y sensibles (Milena, Dainora y Alin, 2008).

En ambos casos, se empleó una guía temática con el fin de abordar los contenidos planteados por el investigador $y$, al mismo tiempo, con la apertura necesaria para incluir elementos emergentes no planificados al momento de la aplicación (Schensul, 2008). Este instrumento fue validado por una investigadora experta en este método como una estrategia de credibilidad para los estudios cualitativos (Cypress, 2017).

\section{Participantes}

Se contó con una muestra total de diez (10) participantes, estudiantes hombres de quinto año de secundaria con una edad aproximada de 16 años $(M=15,89, D E=0,33$ ) de un colegio privado no mixto de Lima Metropolitana. El criterio de selección de los participantes fue el pensamiento crítico manifestado en la vida escolar mediante listas de cotejo de coordinadores y profesores; esto se debe a que los estudiantes críticos se caracterizan por tener una perspectiva autorreflexiva y consciente sobre la cotidianeidad (Ennis, 2011) que 
enriquece el análisis de fenómenos intersubjetivos (Eide, 2008), como es el estudio de las representaciones del estatus. En la selección de participantes se incluyó la saturación temática inductiva, que ocurre cuando ya no emergen códigos nuevos; la saturación temática a priori, momento en que se ejemplifican las categorías; y la saturación de data, que consiste en que la información nueva repite la antigua (Saunders et al., 2018).

\section{Procedimiento}

En primer lugar, se elaboró la guía temática para las entrevistas. A continuación, se seleccionó a los participantes según su pensamiento crítico manifestado en la vida escolar, cruzando las listas de estudiantes elaboradas por miembros de la comunidad escolar. La primera nómina fue propuesta por los tutores y el coordinador del ciclo educativo; la segunda, por un profesor de ciencias sociales; y la última, por el coordinador de lenguaje y el profesor del taller de redacción. Luego, se elaboraron los consentimientos informados que fueron firmados por los padres de los participantes, se reservó la sala de entrevistas de la escuela y se programó su aplicación considerando una fecha y hora en la que los estudiantes pudieran asistir con la autorización de los profesores y autoridades correspondientes.

Una vez finalizada la coordinación de las entrevistas, se procedió a la aplicación de la entrevista de grupo focal con ocho participantes para fomentar la discusión de ideas entre los estudiantes más críticos. Después, se hicieron las entrevistas a profundidad a los dos participantes restantes. En ambos casos, se siguió la estrategia de revisión y verificación de las interpretaciones del investigador con los participantes como una medida para asegurar la credibilidad de los resultados (Cypress, 2017), así como su validez (Noble y Smith, 2015). Cada entrevista duró aproximadamente entre 60 y 70 minutos $(M=64,67, D E=5,03)$. Todas las entrevistas fueron grabadas en archivos de audio y posteriormente transcritas considerando la oralidad del lenguaje y elementos contextuales (Gibbs, 2012).

Luego de la transcripción, se aplicó el análisis temático. La información obtenida se codificó en categorías, incluyendo las planteadas previamente por el investigador (Fereday y Muir-Cochrane, 2006; Fernández, 2006) y aquellas que emergieron en las entrevistas, priorizando las segundas sobre las primeras (Gibbs, 2012). Por último, se elaboraron las posibles relaciones entre las representaciones de los estudiantes con el fin de obtener hallazgos comunes considerando las diferentes narrativas.

\section{RESULTADOS}

Luego del análisis temático y la categorización del contenido, esta sección se compone, principalmente, de fragmentos literales de las entrevistas, como una efectiva técnica para asegurar la validez en los estudios cualitativos (Noble y Smith, 2015). En la primera, 
se desarrollan las estrategias para ganar estatus, mientras que en la segunda se presentan aquellos factores que quitan el estatus en el grupo de estudio.

\section{Estrategias para ganar estatus}

\section{Prestigio}

El primer elemento valorado por el grupo de estudiantes es el éxito académico. Los participantes reconocen que el rendimiento académico tiene marcadores de estatus visibles dentro del sistema escolar: "Creo, en primer lugar, que la persona con el estatus más claro en la promoción es el primer puesto, porque parte de algo que está establecido y se ve, que es el sistema de notas y la posición en la promoción". “Si el primero de la promoción dice que este problema se resuelve de esta manera, nadie lo puede contradecir, porque es el primero; o si hay un ejercicio que es difícil para los primeros puestos, es difícil para todos". Además, "la gente que más se impone nunca va a querer discutir con alguien que es intelectual porque sabe que le pueden voltear la tortilla", lo que implica que las personas dominantes reconocen el estatus de los denominados intelectuales, dado que en una pugna verbal pueden perder valoración social.

Asimismo, los participantes afirman que la conducta prosocial, la amabilidad y el altruismo aumenta el estatus. "Si eres buena gente, eso ayuda"; "claro, los que ayudan a los demás e influyen en el grupo para bien"; "se ganan el respeto porque es también lo que busca el colegio y es reconocido por el grupo en general". Otro participante refuerza esta idea con lo siguiente: "Tú sabes que estas personas son buena gente porque te ayudaron en algún momento con un problema tuyo sin ser tu amigo cercano, y te acuerdas de eso"; es decir, estas conductas son recordadas por el grupo. "Yo creo que se trata de caerles bien a todos. Creo que lo que te da prestigio es que todos se sientan en la capacidad de hablarte, siendo tu amigo o tu compañero, incluso habiéndote peleado con él antes, pero con la seguridad de que sí te va a ayudar, sin importar la situación, el saber que la persona te va a acoger". Las conductas prosociales otorgan prestigio no solo por la aceptación dentro del grupo de estudiantes, sino porque corresponde a los objetivos de la escuela como institución: "Es lo que el colegio busca".

La competencia deportiva es también un factor que aumenta el estatus. "El deporte suma si eres bueno. Si estás en la banca, nunca juegas y eres invisible, es nulo; pero si eres, por ejemplo, una estrella en básquet, vóley o atletismo y ganas medallas, sumas, te da puntos". Otro participante dice lo siguiente: "Creo que el colegio le da mucho valor al deporte, hay reconocimientos y premios, entonces, eso también importa". Por su parte, otro estudiante afirma que "hay deportistas buena gente y otros que molestan a la gente, que son malos e intimidan porque son fuertes". Esto implica que la competencia deportiva da prestigio porque es una actividad socialmente valorada y, en algunos casos, involucra también ciertas cualidades asociadas a la dominancia como la fortaleza física. 
La combinación de estos tres elementos, los más valorados por la promoción, da un mayor prestigio que solo destacar en alguno de ellos. "Si tienes un perfil integral, da un mayor estatus a nivel general, pues eres referente en varios grupos". A lo que otro participante añade que "sí, la gente más valorada es la que hace más de una sola cosa, que destaca en más de una faceta"; "si eres intelectual y eres de los que acoge a los demás, tienes mucho más prestigio, llegas a la gente"; "ese perfil que busca el colegio de persona integral sí pesa mucho".

\section{Dominancia}

Uno de los participantes introdujo, de manera espontánea, la dominancia como estrategia para obtener estatus: "No sé si los factores solos te dan estatus, lo que sí creo que te lo da es la personalidad de dominancia. Por ejemplo, Tomás no es el mejor en notas, no practica deportes, pero siempre sale adelante $y$, a pesar de que no todos estén de acuerdo con él, hace prevalecer su opinión y, aunque no lo tomen en serio, lo que él dice se hace". Otro estudiante narra un caso de toma de decisión grupal: "Me acuerdo de que cuando estábamos viendo la casaca de promoción, al inicio preguntaban quién quería la manga de un color y todos se quedaban con la mano abajo; pero cuando veían que otros levantaban la mano, esos que siempre toman las decisiones, ya todos levantaban". Los participantes, además, afirman que existe un grupo dominante que se caracteriza por amenazar e intimidar al resto, y que es el único cohesionado: "Tienen un nombre, H201, siempre tratan de imponerse en todas las actividades y decisiones de la promoción, te gritan e insultan si los contradices".

Sin embargo, otro participante afirma que "estas personas dominantes, con aquellas personas que tienen una aceptación positiva en toda la promoción, saben que no pueden meterse, saben que con estas personas en particular no van a poder, saben que si se enfrentan a ellos van a perder porque toda la promoción se les va a venir encima". Uno de sus compañeros comenta también que "ellos no son temidos por los inteligentes, porque pueden ser ridiculizados". Afirman, finalmente, que "conforme hemos ido madurando, ya no todos quieren ser como ellos y ni los respetamos como antes"; "ellos se autodecretan como el grupo dominante, cuando no lo son, se asignan un estatus que ya no tienen".

Uno de los miembros del grupo H201 aseveró, por el contrario, que "la gente H201 sigue siendo la más respetada de la promoción, la gente les sigue haciendo caso por miedo y también porque algunos son buena gente, otros son buenos en notas y todos hacen deporte; y que hay un grupo de gente que ha elegido el colegio para cosas de liderazgo, que creen que son los jefes de la promoción, pero en el fondo nadie los respeta". Esto revela que los miembros de este grupo dominante mantienen y actualizan su estatus ya no solo desde la dominancia, sino también desde elementos que confieren prestigio, y que hay cierta pugna con otro grupo de estudiantes que ha ido ganando estatus por esta 
vía. Por último, agrega dos factores presentes en la dominancia: el primero es la asociación de los estudiantes al consumo de sustancias psicoactivas, pues "mucha gente de la promoción piensa que los de mi grupo fuman todo el día y se meten drogas duras, pero nada que ver, es solo la imagen que quieren transmitir para dar miedo"; mientras que el segundo es el "ser sociable y relacionarse con chicas, no con cualquier tipo de gente por si acaso, eso también da estatus".

\section{Elementos que quitan estatus}

En esta categoría, encontramos dos elementos asociados negativamente tanto con la dominancia, pues son signos de debilidad, como con el prestigio, pues constituyen características que no son valoradas por el grupo de estudio. En primer lugar, la identidad sexual no heterosexual hace que los individuos que la posean pierdan estatus. Sobre esto, los participantes concuerdan en que "ser gay sí afecta, puede hacer que incluso muchos te quiten respeto". Sin embargo, los estudiantes sostienen que "el serlo no te quita estatus, sino lo que lo quita es tu actitud disforzada; es más que lo muestre antes que lo sea en sí"; "hay dos tipos de homosexuales, los que son tranquilos, que a ellos nadie los molesta, y los que son llamativos, a los que nadie respeta, los molestan porque la mayoría cree que un gay tiene que estar callado". Estos extractos evidencian la heteronormatividad presente en el grupo, que genera prejuicios ante la diversidad sexual y discriminación contra aquellos sujetos que tengan una expresión de género no masculina (Kjaran, 2017).

El segundo elemento que afecta negativamente el estatus es el padecer de un problema de salud mental que no tenga signos físicos. "Si tienes algún síndrome que no sea síndrome de Down, no te van a respetar". Sobre una situación particular con un estudiante, uno de los participantes comentó que "en tercero no salió tan bien como antes, pero ayudaba a los demás, de ahí se loqueó con algo". Otro añade que "tuvo un cuadro de estrés serio que lo afectó socialmente, porque muchas personas en vez de tomarlo empáticamente lo tomaron de loco". Al respecto, otro estudiante agrega que "algunas personas hasta le tuvieron un poco de odio porque 'esta persona dice tener tal cosa y ya todos le creen', 'no puede ser, pues, o sea, yo me puedo tildar de loco y ya está', porque salía de clases y esas cosas". Incluso, uno de ellos manifestó lo siguiente: "Yo leí algo que escribió el pata y es locazo, algo que nunca te hubieras imaginado; ahora, cuando conversas con él, no es que te das cuenta, pero ya no tiene una postura tan firme, sino que está abierto a todo". Esto revela los prejuicios y la discriminación, el estigma al que son sujetos aquellos estudiantes que tienen trastornos de salud mental que no involucran secuelas físicas (Rojas, 2019). 


\section{DISCUSIÓN}

Luego de analizar los resultados, se puede afirmar que las dos rutas, tanto la dominancia como el prestigio, son efectivas para lograr estatus en la promoción escolar del grupo de estudio (Cheng et al., 2013). La distribución del estatus está en proceso de transición, es decir: de tener una cima conformada por un solo grupo de estudiantes dominantes a una cima compuesta por más de un grupo. En los últimos años de educación secundaria, el prestigio ha ido ganando efectividad como estrategia de ascenso en la jerarquía social frente a la dominancia, que antes era la ruta principal.

Las características más valoradas por el grupo de estudiantes, que otorgan estatus a través del prestigio, son el éxito académico, la conducta prosocial y la competencia deportiva. Los estudiantes que tienen éxito académico, denominados intelectuales, gozan también de un alto estatus en la promoción: su capacidad cognitiva los habilita para resolver problemas grupales y para confrontar exitosamente las agresiones verbales de los estudiantes dominantes. Asimismo, aquellos estudiantes con conductas prosociales indiscriminadas, es decir, que son altruistas y amables con todos los miembros de la promoción, sin importar el grado de cercanía interpersonal o conflictos pasados, son altamente valorados por todo el grupo, ejercen gran influencia y apoyo colectivo. Por último, los estudiantes que son deportistas destacados también poseen un alto estatus porque cuentan con el reconocimiento institucional de la escuela y el de sus pares.

Por otra parte, la dominancia, que se basa en la intimidación y agresividad (Cheng et al., 2013), mantiene su efectividad, como lo demuestran estudios anteriores (Faris y Felmlee, 2009; Faris y Felmlee, 2011). En el presente trabajo, el grupo de estudiantes dominantes se caracteriza por conductas verbalmente agresivas, por la fortaleza física evidenciada en la competencia deportiva y por la asociación con el consumo de drogas. Además, combina su estatus con las habilidades más valoradas que otorgan prestigio, como el éxito académico y la competencia deportiva. Este grupo, conformado por trece estudiantes de la promoción, que tiene nombre propio (H201), es percibido por el resto de pares como altamente cohesionado. Se puede sugerir, igualmente, que el grupo dominante performa una masculinidad sexista, pues esta se caracteriza por la agresión, el autoritarismo y la dominancia social (Garaigordobil y Aliri, 2013).

Considerando lo desarrollado hasta aquí, es preciso señalar que la distribución del estatus no solo está en transición, sino en pugna, principalmente, entre dos grupos. El primero es el dominante, cuyo estatus se basa en conductas impositivas y verbalmente agresivas, en la fortaleza física evidenciada en la competencia deportiva y en la asociación con el consumo de drogas, mientras que el segundo es un grupo de estudiantes que combinan los elementos que dan prestigio (éxito académico, conducta prosocial y competencia deportiva), aquellos que tienen el "perfil integral", que fueron convocados por el colegio para actividades de liderazgo. Los integrantes de ambos grupos afirman 
que los miembros del exogrupo se autoasignan un estatus del que carecen, que nadie les ha reconocido, aseverando que son ellos los que poseen ahora el estatus más alto. Por otra parte, es importante mencionar que la competencia deportiva incluye elementos que pertenecen a factores asociados con el prestigio, como el reconocimiento institucional y de pares, y componentes asociados a la dominancia, como la fortaleza física y la exaltación de la masculinidad (Kopelovich, 2019).

Por último, hay dos factores que quitan estatus y vuelven a un estudiante sujeto de agresión en la vida escolar. El primero es la identidad sexual no heterosexual, particularmente por tratarse de un colegio de hombres, pues la masculinidad hegemónica es necesariamente heterosexual. Sin embargo, el homosexual puede masculinizarse en tanto asuma expresiones de género consideradas como masculinas. En este sentido, mientras el estudiante homosexual se aleje del rol de género masculino estereotipado, y su expresión de género tienda a lo catalogado socialmente como femenino, la devaluación será mayor. Esto revela el preocupante clima heteronormativo de muchas escuelas en Latinoamérica, caracterizado por la marginación y discriminación de la diversidad sexual, específicamente, en relación con las expresiones de género (Rojas, 2019) y la necesidad de componer espacios seguros para la comunidad LGBTQ (Rojas, 2019; Bain y Podmore, 2019; Toomey, McGuire y Russell, 2012).

El segundo elemento es ser diagnosticado con un trastorno mental que no presente signos físicos. Esto lapida socialmente al estudiante, pues, al ser tratado como "loco", es totalmente invalidado como sujeto social frente a todos los miembros de la promoción de estudiantes. El estigma sobre los trastornos de salud mental, muy presente en la región latinoamericana (Mascayano, Lips, Mena y Manchego, 2015), genera incredulidad por la falta de marcadores físicos del problema e incluso hostilidad hacia los beneficios recibidos. Esta preocupante situación revela la urgente necesidad de combatir el estigma sobre los trastornos mentales mediante programas y un currículo comprensivo (Chandra y Minkovitz, 2006; Ke et al., 2014; Milin et al., 2016).

\section{CONCLUSIÓN}

En suma, el estatus social escolar está en proceso de transición y pugna. Ambas estrategias, la dominancia y el prestigio, son efectivas para ganar estatus en el grupo, pero con diferentes alcances. El grupo dominante mantiene su influencia, atención y deferencias en la vida escolar por medio de la intimidación basada en agresiones verbales, fortaleza física y la asociación con el consumo de drogas. El grupo caracterizado por el prestigio, por el contrario, ha ido ganando estatus de manera progresiva definiéndose en oposición al grupo dominante a través del éxito académico, la conducta prosocial y los logros deportivos, apoyado en los reconocimientos institucionales de la escuela en estos rubros. El estatus más bajo lo poseen sujetos de discriminación por tener una identidad sexual 
distinta de la heterosexual relacionada con una expresión de género femenina, así como por el estigma ante el diagnóstico de un trastorno mental que no presenta signos físicos.

\section{REFERENCIAS}

Bain, A., y Podmore, J. (2019). Challenging heteronormativity in suburban high schools through "surplus visibility": gay-straight alliances in the Vancouver city-region, Gender, Place \& Culture. doi: 10.1080/0966369X.2019.1618798

Berg, B. (2008). Qualitative research in social sciences. En L. M. Given (Ed.), The SAGE encyclopedia of qualitative research methods (1. ${ }^{\text {a }}$ ed, pp. 826-831). Thousand Oaks, CA: SAGE Publications.

Berthier, N. (2006). Les techniques d'enquête en sciences sociales. Méthode et exercices. París: Armand Colin.

Bianchi, A., Lancianese, D., y Hunter, V. (2006). Gifts and social status: on-going experimental tests of behavior-status and social exchange theories. Conference Papers. American Sociological Association, 1.

Chandra, A., y Minkovitz, C. S. (2006). Factors that influence mental health stigma among 8th grade adolescents. Journal of Youth and Adolescence, 36(6), 763-774. doi: $10.1007 / \mathrm{s} 10964-006-9091-0$

Cheng, J. T., Tracy, J. L., Foulsham, T., Kingstone, A., y Henrich, J. (2013). Two ways to the top: evidence that dominance and prestige are distinct yet viable avenues to social rank and influence. Journal of Personality and Social Psychology, 104(1), 103-125. https://doi.org/10.1037/a0030398

Cypress, B. S. (2017). Rigor or reliability and validity in qualitative research: perspectives, strategies, reconceptualization, and recommendations. Dimensions of Critical Care Nursing, 36(4), 253-263. https://doi.org/10.1097/DCC.0000000000000253

Dilshad, R. M., y Latif, M. I. (2013). Focus group interview as a tool for qualitative research: an analysis. Pakistan Journal of Social Sciences (PJSS), 33(1), 191-198.

Eide, P. (2008). Recruiting participants. En L. M. Given (Ed.), The SAGE encyclopedia of

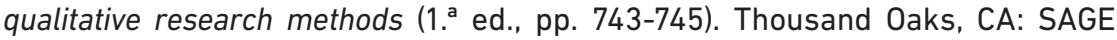
Publications.

Ennis, R. (2011). The nature of critical thinking: an out line of critical thinking dispositions and abilities. Recuperado de https://education.illinois.edu/docs/default-source/ faculty-documents/robert-ennis/thenatureofcriticalthinking_51711_000. pdf?sfvrsn=7bb51288_2

Faris, R., y Felmlee, D. (2009). Mean girls in the middle: network centrality, gender, and bullying in adolescence. Conference Papers. American Sociological Association, 1. 
Faris, R., y Felmlee, D. (2011). Status struggles: network centrality and gender segregation in same and cross-gender aggression. American Sociological Review, 76(1), 48-73. https://doi.org/10.1177/0003122410396196

Fereday, J., y Muir-Cochrane, E. (2006). Demonstrating rigor using thematic analysis: a hybrid approach of inductive and deductive coding and theme development. International Journal of Qualitative Methods, 5(1), 80-92. https://doi.org/10.1177/ 160940690600500107

Fernández, L. (2006). ¿Cómo analizar datos cualitativos? Butlleti LaRecerca, 7(1), 1-13.

Fiske, S. T., Dupree, C. H., Nicolas, G., y Swencionis, J. K. (2016). Status, power, and intergroup relations: the personal is the societal. Current Opinion in Psychology, 11, 44-48. doi:10.1016/j.copsyc.2016.05.012

Garaigordobil, M., y Aliri, J. (2013). Relaciones del sexismo con justificación de la violencia, y con otras formas de prejuicio como la dominancia social y el autoritarismo. Estudios de Psicología, 34(2), 127-139. doi: 10.1174/021093913806751384

Gibbs, G. (2012). El análisis de datos cualitativos en investigación cualitativa. Madrid: Morata.

Henrich, J., y Gil-White, F. J. (2001). The evolution of prestige: freely conferred deference as a mechanism for enhancing the benefits of cultural transmission. Evolution and Human Behavior, 22(3), 165-196. doi:10.1016/s1090-5138(00)00071-4

Henry, S. E. (2013). Bodies at home and at school: toward a theory of embodied social class status. Educational Theory, 63(1), 1-16. doi: 10.1111/edth.12006

Hernández, R., Fernández, C., y Baptista, P. (2014). Metodología de la investigación (6. a ed.). México D. F.: McGraw-Hill.

Kahneman, D. (2013). Thinking, fast and slow. Nueva York, NY: Farrar, Straus and Giroux.

Kakkar, H., y Sivanathan, N. (2017). When the appeal of a dominant leader is greater than a prestige leader. Proceedings of the National Academy of Sciences, 114(26), 67346739. doi: $10.1073 /$ pnas.1617711114

Kakkar, H., Sivanathan, N., y Gobel, M. (2019). Fall from grace: the role of dominance and prestige in the punishment of high-status actors. Academy of Management Journal, 63(2). doi: 10.5465/amj.2017.0729

Ke, S., Lai, J., Sun, T., Yang, M. M. H., Wang, J. C. C., y Austin, J. (2014). Healthy young minds: the effects of a 1-hour classroom workshop on mental illness stigma in high school students. Community Mental Health Journal, 51(3), 329-337. doi: 10.1007/s10597-014-9763-2

Kjaran, J. I. (2017). The institutionalization of heteronormativity in schools. En Constructing sexualities and gendered bodies in school spaces (pp. 97-146). Nueva York, NY: Palgrave Macmillan. 
Kopelovich, P. (2019). Fútbol como práctica de exaltación de masculinidades: el caso de un colegio secundario de sectores medios de la provincia de Buenos Aires, Argentina. Cuadernos de la Facultad de Humanidades y Ciencias Sociales. Universidad Nacional de Jujuy, 55. Recuperado de http://sedici.unlp.edu.ar/ handle/10915/90119

Kornblit, A., Verardi, M., y Beltramino, F. (2009). El uso de las metodologías cualitativas en los estudios sobre drogadicción. En A. Merlino (Coord.), Investigación cualitativa en ciencias sociales (pp. 85-108). Buenos Aires: Cengage Learning.

Mascayano, F., Lips, W., Mena, C., y Manchego, C. (2015). Estigma hacia los trastornos mentales: características e intervenciones. Salud Mental, 38(1), 53-58.

Mattan, B. D., Kubota, J. T., y Cloutier, J. (2017). How social status shapes person perception and evaluation: a social neuroscience perspective. Perspectives on Psychological Science, 12(3), 468-507. https://doi.org/10.1177/1745691616677828

Milena, Z. R., Dainora, G., y Alin, S. (2008). Qualitative research methods: a comparison between focus-group and in-depth interview. Annals of the University of Oradea, Economic Science Series, 17(4), 1279-1283.

Milin, R., Kutcher, S., Lewis, S. P., Walker, S., Wei, Y., Ferrill, N., y Armstrong, M. A. (2016). Impact of a mental health curriculum on knowledge and stigma among high school students: a randomized controlled trial. Journal of the American Academy of Child \& Adolescent Psychiatry, 55(5), 383-391.e1. doi: 10.1016/j.jaac.2016.02.018

Morgan, D. (2012). Focus group and social interaction. En J. F. Gubrium, J. A. Holstein, A. B. Marvasti y K. D. McKinney (Eds.), The SAGE handbook of interview research (pp. 161-176). Thousand Oaks, CA: SAGE Publications.

Noble, H., y Smith, J. (2015). Issues of validity and reliability in qualitative research. Evidence Based Nursing, 18(2), 34-35.

Panchal, S., y Gill, T. (2019). When size does matter: dominance versus prestige based status signaling. Journal of Business Research. https://doi.org/10.1016/j. jbusres.2019.03.047

Pitt, D. (2012). Mental representation. En E. N. Zalta (Ed.), The Stanford encyclopedia of philosophy. Recuperado de https://plato.stanford.edu/cgi-bin/encyclopedia/ archinfo.cgi?entry=mental-representation

Rahal, D., Chiang, J. J., Bower, J. E., Irwin, M. R., Venkatraman, J., y Fuligni, A. J. (2019). Subjective social status and stress responsivity in late adolescence. Stress, 1-10. doi: 10.1080/10253890.2019.1626369

Redhead, D. J., Cheng, J. T., Driver, C., Foulsham, T., y O'Gorman, R. (2018). On the dynamics of social hierarchy: a longitudinal investigation of the rise and fall of prestige, dominance, and social rank in naturalistic task groups. Evolution and Human Behavior, 40(2). doi: 10.1016/j.evolhumbehav.2018.12.001 
Roberts, A., Palermo, R., y Visser, T. A. W. (2019). Effects of dominance and prestige based social status on competition for attentional resources. Scientific Reports, 9, 2473. https://doi.org/10.1038/s41598-019-39223-0

Rojas, E. (2019). Heteronormatividad escolar en México: reflexiones acerca de la vigilancia y castigo de la homosexualidad en la escuela. Sexualidad, Salud y Sociedad, 33, 180-199. https://doi.org/10.1590/1984-6487.sess.2019.33.10.a

Saunders, B., Sim, J., Kingstone, T., Baker, S., Waterfield, J., Bartlam, B., Bourroughts, H., y Jinks, C. (2018). Saturation in qualitative research: exploring its conceptualization and operationalization. Quality \& Quantity, 52(4), 1893-1907. doi: 10.1007/ s11135-017-0574-8

Schensul, J. (2008). Methodology. En L. M. Given (Ed.), The SAGE encyclopedia of qualitative

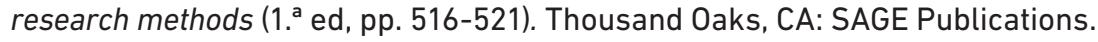

Searle, J. (1995). The construction of social reality. Londres: Penguin Philosophy.

Sweeting, H., y Hunt, K. (2014). Adolescent socio-economic and school-based social status, health and well-being. Social Science \& Medicine, 121, 39-47. doi: 10.1016/j. socscimed.2014.09.037

Toomey, R. B., McGuire, J. K., y Russell, S. T. (2012). Heteronormativity, school climates, and perceived safety for gender nonconforming peers. Journal of Adolescence, 35(1), 187-196. doi: 10.1016/j.adolescence.2011.03.001

Vieytes, R. (2009). Campos de aplicación y decisiones de diseño en la investigación cualitativa. En A. Merlino (Coord.), Investigación cualitativa en ciencias sociales (pp. 43-83). Buenos Aires: Cengage Learning.

Witkower, Z., Tracy, J. L., Cheng, J. T., y Henrich, J. (2020). Two signals of social rank: Prestige and dominance are associated with distinct nonverbal displays. Journal of Personality and Social Psychology, 118(1), 89-120. https://doi.org/10.1037/ pspi0000181 